\title{
Convection and pulsation in red giant stars
}

\author{
P. R. Wood \\ Research School of Astronomy \& Astrophysics, Australian National University, Cotter Road, \\ Weston Creek ACT 2611, Australia \\ email: wood@mso.anu.edu.au
}

\begin{abstract}
Convection strongly influences the periods, stability and amplitudes of pulsation in red giant stars. For example, changing the efficiency of convection (the mixing length parameter) changes the radius and the effective temperature of a red giant, which in turn changes the pulsation period at a given luminosity. Since essentially all the energy flux outside the nuclear-burning core is carried by convection, it is the variation of convective energy transport throughout the pulsation cycle that determines pulsation stability. In both linear and nonlinear pulsation models, the turbulent viscosity that results from the interaction of pulsation with turbulent convection provides a strong damping effect on pulsation and, in nonlinear models, it determines the limiting amplitude. In this paper, these and other topics are discussed.
\end{abstract}

Keywords. Stars: AGB and post-AGB, stars: oscillations, stars: late-type

\section{Introduction}

The pulsation theory of red giant stars is still very inexact compared to similar theories for warmer variables such as Cepheids and RR Lyraes. The main reason for our poor understanding of variable red giants is that convection dominates energy transport in the envelope, the part of the star to which the pulsation is confined. Observationally, the long periods (up to several years) of these stars mean that a lot of patience is required in order to study the pulsation cycle: fortunately, this problem has been remedied in recent years, at least for simple photometric observations, by the long-term microlensing surveys such as MACHO and OGLE. These have revealed a wealth of new detail regarding pulsating red giants, especially those in the Magelllanic Clouds where all the observed stars are at essentially the same, known distance.

\section{Current observational data}

Over the last 10 years, there have been many detailed studies of the variable red giants found in the microlensing surveys (Wood et al. 1999; Wood 2000; Ita et al. 2004; Soszyński et al. 2004a; Soszyński et al. 2005; Fraser et al. 2005). The most remarkable feature of these studies is the discovery that the red giant variables lie on six separate sequences, originally labelled A,...,E by Wood et al. (1999), although the B sequenec has since been shown to split into two, close, parallel sequences (Ita et al. 2004; Fraser et al. 2005). An example of the $(K, \log P)$ diagram for red giant variables is shown in Figure 1. The sequences A, B and C correspond to stellar pulsation in low order radial modes (Wood et al. 1999), sequence D is of unknown origin (Hinkle et al. 2002; Wood et al. 2004), while sequence E consists of binary stars (Wood et al. 1999; Soszyński et al. 2004b). Ita et al. (2004) note that the lower and upper parts of sequences A and B are slightly offset: this is almost certainly due to the the fact that the lower and upper parts of these sequences correspond to first giant branch and AGB stars, respectively, pulsating in the same mode but with different core masses as a result of the change in evolutionary 


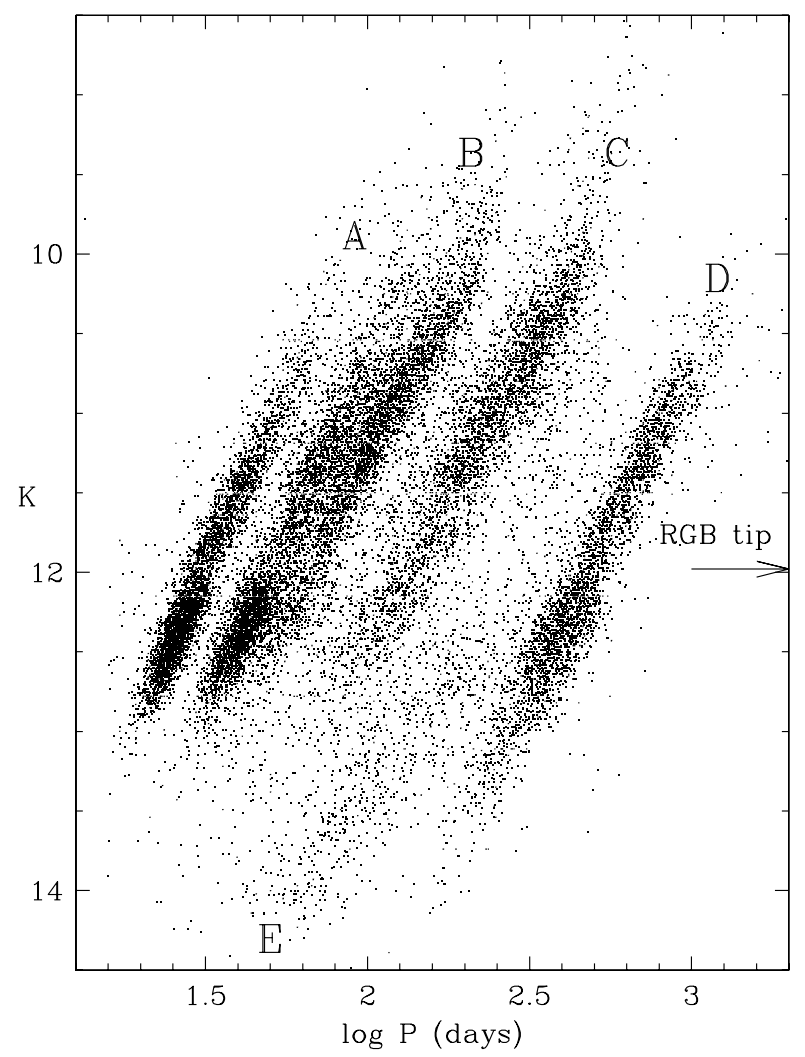

Figure 1. $K$ plotted against $\log P$ for red variables in the LMC using data similar to that in Fraser et al. (2005). The separate sequences are labelled with the original Wood et al. (1999) letters: clearly, sequence B consists of two closely-spaced sequences.

phase. Some examples of the light curves of variable red giants on the various sequences are shown in Wood et al. (1999).

From a theoretical point of view, the PL diagrams discussed above can provide a constraint on the stellar radius and mass via the $P-M-R$ relation of stellar pulsation. This is discussed in Section 3. A feature of the light curves of red giants is that multiple modes are often present. This allows the determination of period ratios as well as individual mode periods (Wood et al. 1999; Soszyński et al. 2004a). A plot of period ratio against period reveals information about the mass distribution throughout the convective envelope, and hence it can in principle provide information about the efficiency of convective energy transport at various radii.

The PL diagrams for red giants such as Figure 1 show the regions in luminosity where various pulsation modes are unstable. Since convective energy transport largely determines the pulsational stability of a red giant, the region where variables are seen in the red giant PL relations can provide some constraints on convection theory. Even better, in star clusters, the position of unstable modes is more clearly defined (see the 47 Tuc PL relations in Lebzelter \& Wood 2005), the mass is known, and a more stringent constraint can be placed on the convective energy transport theory. 


\section{Linear pulsation models}

Linear, non-adiabatic pulsation models give reasonable approximations for the pulsation period (but see Section 5) and they show whether a model is pulsationally unstable or not. Existing linear pulsation models for red giants assume spherical symmetry, no magnetic fields or rotation, and they use a simple mixing-length theory of convection (Fox \& Wood 1982; Ostlei \& Cox 1986), or a higher order extension including turbulent pressure (Ostlei \& Cox 1986; Gautschy 1999), or a detailed convection-pulsation interaction involving turbulent viscosity (Xiong et al. 1998). Both the mixing-length theory and the higher order theories involve a number of constants (free parameters) that must be set by comparison with observational data or by general physical reasoning.

The periods of red giant pulsation have not so far been used to constrain the parameters of the convection theories. In practice, with simple convective theories like the mixinglength theory (MLT), the radius is determined by the mixing-length $\ell$, which can be set by the requirement that the giant branch has the observed $T_{\text {eff }}$. Then, for field stars such as those in the field of the Magellanic Clouds, the stellar mass can be determined. This was done for the Magellanic Cloud variables in the MACHO database by Wood et al. (1999), and for the red variables in 47 Tuc by Lebzelter \& Wood (2005). In the latter case, the derived masses gave direct evidence that mass loss has caused a reduction of stellar mass. In principle, the (period ratio, P) relations could be used to provide some constraint, but this has not been done so far.

The comparison of model stability with observations of red giant stability will provide more stringent constraints on convection theories. In general, it is found that models become more unstable as the luminosity increases or $T_{\text {eff }}$ decreases. Since real red giants obey a $\left(L, T_{\text {eff }}\right)$ relation (they fall on the Hayashi track), there is not really an "instability strip" for red giants as for Cepheids, just a minimum luminosity for instability. Gautschy (1999) has made some attempt to compare observed variable red giant parameters with model calculations, although this did not lead to any real constraint on the convection parameters. The most mathematically detailed stability calculations are those of Xiong et al. (1998). These models suggest that turbulent viscosity may stabilize modes higher than the fourth, as indeed seems to be observed: there is an absence of any PL sequence at periods shorter than sequence-A in Figure 1. However, the absence of higher-order modes may also be because the frequency of these modes is higher than the acoustic cut-off frequency (Wood 2006). Xiong et al. (1998) also find that their models become stable at low effective temperatures, while observationally the coolest, longest period red giants tend to have the largest amplitudes (e.g. Wood et al. 1998). One can conclude that at this stage, although the models generally predict pulsation in the red giant region, current convection theories do not lead to reliable quantitative predictions regarding model stability.

\section{Oscillatory radial modes associated with convection}

While looking for an explanation for the sequence-D modes in Figure 1, Wood (2000b) found a family of oscillatory radial modes that depend explicitly on the interaction of convection with radial oscillation in red giants. These modes are essentially thermal modes since the dynamical term in the momentum equation is insignificant in the determination of the mode characteristics. The mode periods tend to be longer than $\sim 1000$ days (as required for sequence-D modes) but the damping of the modes is enormous. For an $\exp (\omega t)$ time dependence, the (negative) real part of $\omega$ is larger than the imaginary part, at least for the very simple MLT theory used in the calculations. In this situation, it is 


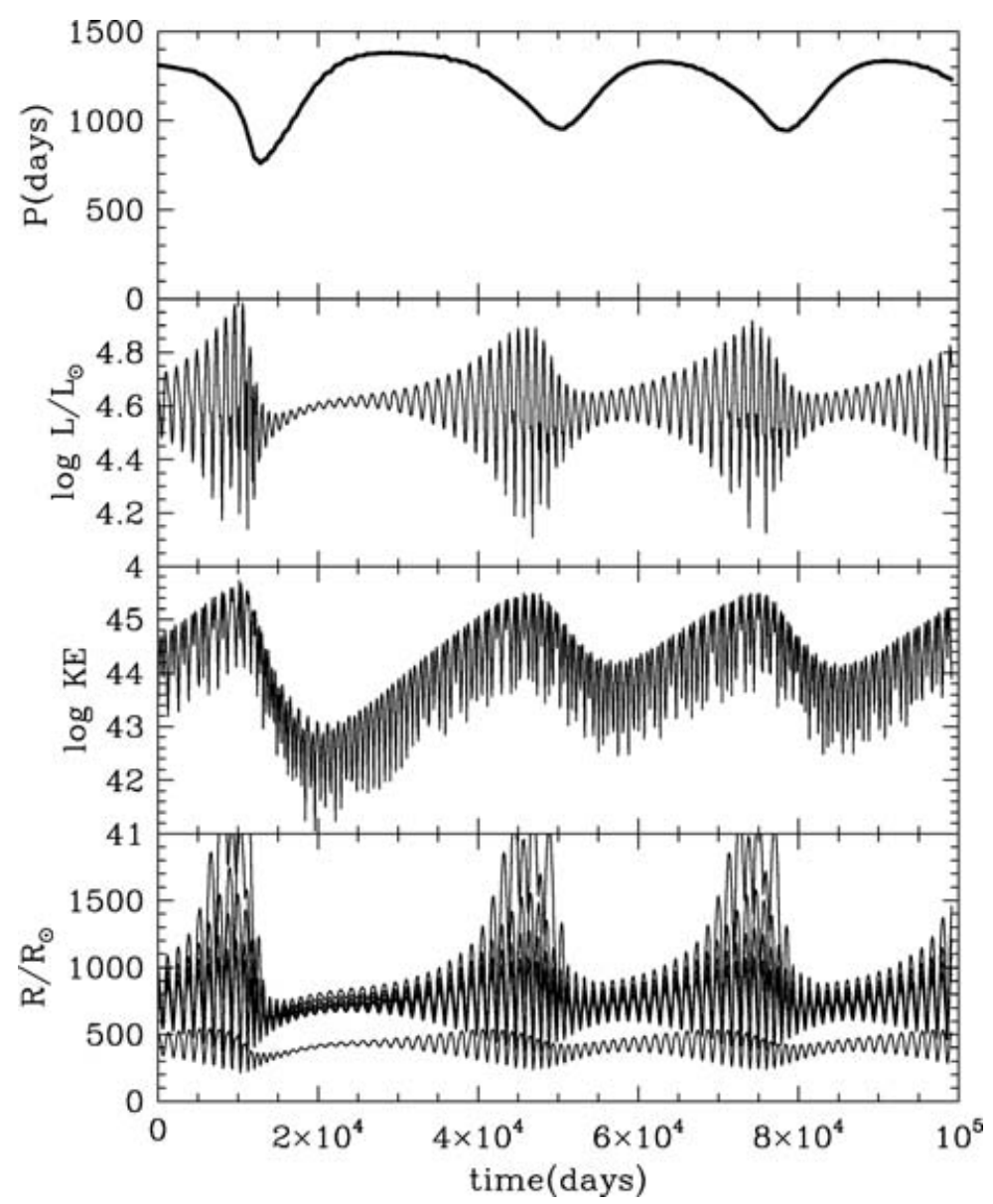

Figure 2. Various quantities plotted against time for a pulsating red giant with $M=5 \mathrm{M}_{\odot}$, $L=41840 \mathrm{~L}_{\odot}$ and metallicity $\mathrm{Z}=0.004$. The top panel shows the period, the second panel shows the surface luminosity, the third panel shows the kinetic energy of pulsation, and the bottom panel shows the radius of a selection of mass zones.

unlikely that these modes would ever play a significant role in the variability of real red giants.

\section{Nonlinear pulsation models}

A complete nonlinear convective model of the outer envelope of a red giant would allow the study of both pulsation and convective energy transport. One or two exploratory calculations of this kind, with many simplifying approximations, have been tried (Freytag et al. 1999; Woodward et al. www.lcse.umn.edu/research/RedGiant/). These studies do indeed find that pulsation occurs, and that significant nonspherical temperature distributions occur as a result of the convective motions. However, with current computational capabilities, systematic studies of the interaction of nonlinear pulsation and convection in red giants has to be confined to spherical geometry and simple convection theories such as the MLT.

A long-standing problem with the modelling of the nonlinear pulsatiom of red giant stars is that the pulsation of the models is driven much too strongly (e.g. Wood 1974; 
Tuchman et al. 1978). A solution to this problem has recently been found with the introduction of turbulent viscosity, a property that results explicitly from the interaction of pulsation with turbulent convection. This turbulent viscosity always has a damping effect (Xiong et al. 1998; Olivier \& Wood 2005). A possibly unsatisfactory aspect of the treatment of turbulent viscosity is that its magnitude is governed by a parameter that can not be easily estimated from theory: its magnitude is adjusted to give the correct pulsation amplitude.

Another important aspect of the interaction of convection with nonlinear pulsation is that this interaction causes the mean structure of the envelope in a large amplitude pulsator to differ from the structure of the static parent star. This means that the nonlinear, nonadiabatic pulsation period (for example, that in a Mira variable) will differ from the linear, nonadiabatic period of the non-pulsating star, or from the period of the star if it was pulsating at small amplitude (Ya'Ari \& Tuchman 1996; Wood 1995; Lebzelter $\&$ Wood 2005). In some of these calculations, the nonlinear period was longer than the linear period, while the reverse was true in other cases. The amplitude and direction of the period change seems to depend on the envelope mass, but no systematic investigation has yet been done.

An extreme example of the interaction of pulsation and convection is shown in Figure 2. In this case, energy is cyclicly fed from pulsation (kinetic energy) to the envelope (thermal and gravitational potential energy) and back again. At the same time, parts of the inner envelope change between convective to radiative energy transport. Perhaps this behaviour is related to the behaviour of the small group of pulsating red giants such as $\mathrm{R}$ Hya and $\mathrm{R}$ Dor that have changing periods and cyclic mode switching (Wood \& Zarro 1981; Bedding et al. 1998; Zijlstra et al. 2002).

\section{References}

Bedding, T.R., Zijlstra, A.A., Jones, A., \& Foster, G. 1998, MNRAS 301, 1073

Fox, M.W. \& Wood, P.R. 1982, ApJ 259, 198

Fraser, O.J., Hawley, S.L., Cook, K.H., \& Keller, S.C. 2005, AJ 129, 768

Gautschy, A. 1999, A\&A 349, 209

Groenewegen, M.A.T. 2004, A\&A 425, 595

Hinkle, K.H., Lebzelter, T., Joyce, R.R., \& Fekel, F.C. 2002, AJ 123, 1002

Ita, Y., Tanabé, T, Matsunaga, N., Nakajima, Y., Nagashima, C., Nagayama, T., Kato, D., Kurita, M., Nagata, T., Sato, S., Tamura, M., Nakaya, H., \& Nakada, Y. 2004, MNRAS 347,720

Lebzelter, T. \& Wood, P.R. 2005, A\&SA 441, 1117

Olivier, E.A. \& Wood, P.R. 2005, MNRAS 362, 1396

Ostlei, D.A. \& Cox, A.N. 1986, ApJ 311, 864

Soszyński, I., Udalski, A., Kubiak, M., Szymański, M., Pietrzyński, G., Zebruń, K., Szewczyk, O., \& Wyrzykowski, L. 2004a, Acta Astr. 54, 129

Soszyński, I., Udalski, A., Kubiak, M., Szymański, M., Pietrzyński, G., Zebruń K., Szewczyk, O., Wyrzykowski, L., \& Dziembowski, W.A. 2004b, Acta Astr. 54, 347

Soszyński, I., Udalski, A., Kubiak, M., Szymański, M., Pietrzyński, G., Zebruń, K., Szewczyk, O., Wyrzykowski, L., \& Ulaczyk, K. 2005, Acta Astr. 55, 331

Tuchman, Y., Sack, N., \& Barkat, Z. 1978, ApJ 219, 183

Wood, P.R. 1974, ApJ 190, 609

Wood, P.R. \& Zarro, D.M. 1981, ApJ 247, 247

Wood, P.R. 1995, in Astrophysical Applications of Stellar Pulsation Theory, ASP Conf. Ser. 83, 127

Wood, P.R., Habing, H.J., \& McGregor, P.J. 1998, A\&̋A 336, 925

Wood, P.R. and the MACHO Collaboration 1999, in IAU Symp. 191 AGB stars, p. 151 
Wood, P.R. 2000b, PASA 17, 18

Wood, P.R. 2000b, in The Impact of Large-Scale Surveys on Pulsating Star research, ASP Conf. Ser. 203, 379

Wood, P.R., Olivier, E.A., \& Kawaler, S.D. 2004, ApJ 604, 800

Wood, P.R. 2006, MmSAI 77, 76

Xiong, D. R., Deng, L., \& Cheng, Q. L. 1998, ApJ 499, 355

Ya'Ari, A. \& Tuchman, Y. 1996, ApJ 456, 350

Zijlstra, A.A., Bedding, T.R., Mattei, J.A. 2002, MNRAS 334, 498

\section{Discussion}

KUPKA: Just a comment: one should not forget that the simulations of Porter and Woodward that you mentioned are fairly idealized (perfect gas equation, radiative transfer, etc). 